\title{
Inherent modulation: a fast chopping method for nulling interferometry
}

\author{
Olivier Absil $^{a}$, Anders Karlsson ${ }^{b}$ and Lisa Kaltenegger ${ }^{b}$ \\ ${ }^{a}$ IAGL, University of Liège, B5C, 17 Allée du 6 Août, B-4000 Sart-Tilman, Belgium \\ ${ }^{b}$ ESA-ESTEC, postbus 299, NL-2200AG Noordwijk, The Netherlands
}

\begin{abstract}
The reduction of the thermal background emission from the local and exozodiacal dust clouds is a critical element for the success of ESA's space mission, DARWIN. Internal modulation, a technique using fast signal chopping, isolating the planetary signal from these noise sources, was proposed by Mennesson and Léger. ${ }^{1}$ In this paper, a short review of internal modulation is given, and new configurations with internal modulation are proposed to reduce the complexity of the beam-combining optics. A modification to the implementation of internal modulation is then investigated. It provides similar performance with a single detector and a greatly simplified optical layout: the number of beam-combiners is reduced by a factor of about two. The principle of inherent modulation is different from internal modulation in that no sub-interferometers are used: different phase shifts are applied to the input beams before recombination such that an asymmetric transmission map is obtained directly, without $\pm \pi / 2$ modulation as used in internal modulation. By combining the phase shifts and the input beams differently a transposed transmission map is obtained, allowing the signal to be chopped. During operations, multiplexing between the two interferometers is performed, such that at any time only one interferometer is being used.
\end{abstract}

Keywords: Nulling space interferometer, direct planet detection, infrared, thermal background, internal modulation

\section{INTRODUCTION}

Darwin is one of the most challenging space mission ever considered by the European Space Agency (ESA). Its main objectives are to detect Earth-like planets orbiting nearby stars and to characterize their atmospheres by means of low resolution spectroscopy (Léger et al. ${ }^{2}$ ). The two difficulties associated with direct planet detection are the huge contrast between the planet and the star and their small angular distance. The flux ratio is typically of $10^{9}$ in the visible and of $10^{6}$ in the mid-infrared, while the angular separation is of 100 mas for a Sun-Earth couple located at 10 parsecs. Infrared nulling interferometry proposed by R. Bracewell ${ }^{3}$ in 1978 , has been selected by ESA as a baseline for the Darwin mission. This technique achieves both a high angular resolution and a high dynamic range by adjusting the phases of the beams coming from various telescopes to produce a fully destructive interference on the optical axis. Darwin will operate in a wavelength band between 6 and 18 microns where suitable tracers for Earth-type atmospheres can be found.

The current mission-model baseline consists of six free-flying $1.5 \mathrm{~m}$ telescopes, arranged in a hexagonal configuration about $50 \mathrm{~m}$ wide, with in the centre of the array a beam-combining satellite equipped with optical benches. This configuration uses a technique called internal modulation which performs fast signal chopping to isolate the planetary signal from other sources. The main drawback of this configuration is the complexity of the recombination optics: it requires six beam-splitters, twelve beam-combiners and nine achromatic phase shifters. Moreover, six single-pixel detectors are needed to ensure a maximum efficiency. In this paper, we propose new configurations to achieve fast signal chopping with a greatly simplified recombination scheme.

Further author information: (Send correspondence to O.A.)

O.A.: E-mail: absil@astro.ulg.ac.be, Telephone: +32-4-366 9761

A.K.: E-mail: Anders.Karlsson@esa.int, Telephone: +31-71-565 3532

L.K.: E-mail: Lisa.Kaltenegger@esa.int 


\section{THE NEED FOR MODULATION}

A nulling interferometer formed of $n$ telescopes with polar coordinates $\left(L_{k}, \delta_{k}\right)$ is characterized by its intensity response $R(\theta, \phi)$, the square modulus of the complex electrical field response $\mathbf{E}(\theta, \phi)$, where $(\theta, \phi)$ are the polar coordinates in the plane of the sky with respect to the optical axis (see Fig. 1). Its expression has been derived by Mennesson ${ }^{4}$ :

$$
R(\theta, \phi)=|\mathbf{E}(\theta, \phi)|^{2}=\left|\sum_{k=1}^{n} A_{k} e^{i 2 \pi\left(L_{k} \theta / \lambda\right) \cos \left(\delta_{k}-\phi\right)} e^{i \phi_{k}}\right|^{2},
$$

where $A_{k}$ and $\phi_{k}$ are the amplitude modulus and phase shift for telescope $k$. The amplitude coefficients are normalized so that for the larger telescope we have $A_{i}=1$ if the whole collected beam is used in the recombination scheme, taking into account the possible losses due to unused beam-combiner outputs. The intensity response projected on the plane of the sky forms the so-called transmission map of the interferometer. This map defines which parts of the field-of-view are transmitted by constructive interference, and which are blocked by destructive interference. No image is formed: we only measure the integrated flux transmitted by the interference pattern on a single pixel detector. Therefore, there is a priori no distinction between the planetary signal and other sources in the field-of-view, such as dust, stellar leakage or thermal background. This problem can be solved either by reducing the amount of spurious signals well below the planetary signal, or by extracting the planetary signal by an appropriate technique.

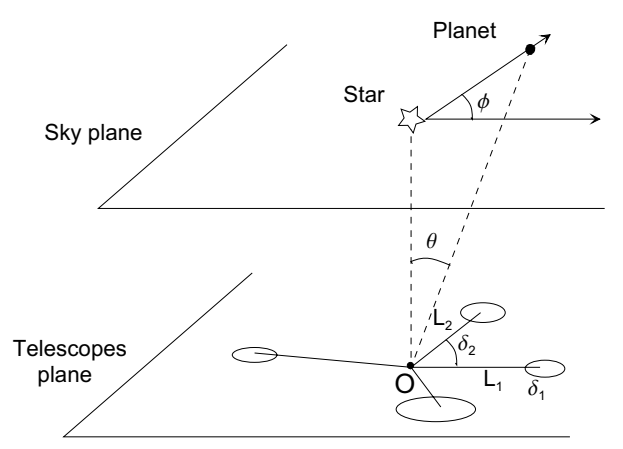

Figure 1. Geometrical configuration of the array and the stellar system. Planetary coordinates are given by the off-axis angular direction $\vec{\theta}=(\theta, \phi)$. Each telescope of index $k$ is located by means of the vector $\overrightarrow{L_{k}}=\left(L_{k}, \delta_{k}\right)$ in a reference frame with arbitrary origin $\mathrm{O}$, used as a common phase reference.

The first requirement for a nulling interferometer is thus to achieve a deep central null, in order to extinguish the stellar light by a factor of $10^{5}$ or more. Mennesson and Mariotti ${ }^{5}$ have shown that, for the target stars and interferometric baselines considered here, this condition can only be achieved if the central transmission of the interferometer is proportional to $\theta^{4}$ (or even better $\theta^{6}$ ), where $\theta$ is the off-axis angle when pointing towards the star. This requires at least three telescopes in a linear arrangement, or four telescopes in a two-dimensional configuration as proven by Absil. ${ }^{6}$

Once the stellar light has been properly cancelled, there are other spurious signals to suppress. These come from the thermal emission of the zodiacal and exozodiacal dust, and from the interferometer itself. This is the reason why Bracewell proposed to rotate the interferometer around its line-of-sight with a period of about one hour. The planetary flux is then modulated as the planet crosses bright and dark fringes in the transmission map, so that it can be retrieved by synchronous demodulation. However, a low-frequency rotation of the interferometer is not enough to completely cancel these spurious signals, as proven below.

- Zodiacal Dust Cloud. The zodiacal cloud is a tenuous disk that surrounds the Sun, composed of dust released from active comets and colliding asteroids. It is heated to about $300 \mathrm{~K}$ at 1 A.U. from the Sun, and is a major contributor to the infrared background seen by Darwin: the total zodiacal flux collected by $1 \mathrm{~m}$-class telescopes is about 1000 times larger than the flux from the exoplanet. This signal is not 
modulated by rotation of the array, so that it can be canceled by signal processing. However, the real problem comes from its associated photon noise, and above all from the long-term fluctuations of its brightness. These fluctuations can be confused with the slowly modulated flux of the planet unless fast signal chopping is used.

- Exozodiacal Dust Cloud. Another major source of noise is the exozodiacal cloud surrounding the target star. Considering a dust cloud similar to ours, its integrated flux over the first 5 A.U. is about 300 times larger than the planetary emission at $10 \mu \mathrm{m}$. The main problem with the exozodiacal cloud is that its emission is also modulated by rotation of the array (unless it is seen face-on), so that it can be confused with the exoplanet.

- Instrumental Background. In order to keep the temperature of the cold optics of Darwin as low as possible, a planar interferometer concept is required. This avoids a strong thermal coupling between the different telescopes. The optical elements will be passively cooled to $40 \mathrm{~K}$, while the baseline detectors require active cooling to $6 \mathrm{~K}$. The thermal emission of the instrument can generate troublesome levels of background signal if its temperature exceed $40 \mathrm{~K}$. Moreover, fluctuations of temperature and emissivity in the optical path to the detector will induce fluctuations of the thermal background levels, which have to be monitored at a high enough frequency.

Therefore, there are two other major requirements for a nulling interferometer: first, to sample the background fluctuations at a high enough frequency, and second, to discriminate between the planet and the exozodiacal cloud. The first attempts to extract the planetary signal from the exozodiacal emission were made by Angel and Woolf ${ }^{7}$ who proposed to use the strong modulation of the exozodiacal emission at twice the interferometer rotation frequency due to its symmetry, and by Mennesson and Mariotti ${ }^{5}$ who proposed to break the central symmetry of the interferometer, for example by using five telescopes regularly located on a circle or on an ellipse. But these configurations did not achieve the required high frequency modulation, and thus were still sensitive to background fluctuations and to other $1 / f^{\alpha}$ type noises. This is the reason why internal modulation has been proposed by Mennesson and Léger, ${ }^{1}$ following an original idea of J.-M. Mariotti.

\section{INTERNAL MODULATION}

The principle of internal modulation is to divide a telescope array into two (or more) nulling interferometers and to recombine their outputs with a time variable phase shift in order to achieve a fast modulation of the planetary signal (Mennesson and Léger ${ }^{1}$ ). The sub-interferometers are formed by dividing the amplitudes of the light beams collected by each telescope (Fig. 2a-b). Both have a real entrance pupil, which means that the phase shifts applied to their light beams are restricted to 0 or $\pi$. Their transmission maps have different shapes (Fig. 2c), but both achieve a $\theta^{4}$ central transmission.

The outputs of the two sub-interferometers are then recombined on a loss-less beam combiner, which induces a $\pi / 2$ phase shift between the input beams in the first output, and a $-\pi / 2$ phase shift in the second output. The new transmission maps associated to these outputs are both asymmetric, although symmetric to each other with respect to their center (Fig. $2 \mathrm{~d}$ ). Denoting $R_{12}(\theta, \phi)$ and $R_{21}(\theta, \phi)$ the intensity responses associated to the two new outputs, this property writes $R_{12}(\theta, \phi+\pi)=R_{21}(\theta, \phi)$. This can easily be proven by developing the expression of the two intensity responses, which write:

$$
\begin{gathered}
R_{12}(\theta, \phi)=\frac{1}{2}\left|\mathbf{E}_{1}(\theta, \phi)+e^{i \pi / 2} \mathbf{E}_{2}(\theta, \phi)\right|^{2}, \\
R_{21}(\theta, \phi)=\frac{1}{2}\left|\mathbf{E}_{1}(\theta, \phi)+e^{-i \pi / 2} \mathbf{E}_{2}(\theta, \phi)\right|^{2} .
\end{gathered}
$$

By developing the square modulus, we get:

$$
\begin{aligned}
R_{12}(\theta, \phi) & =\frac{1}{2}\left|\mathbf{E}_{1}(\theta, \phi)\right|^{2}+\frac{1}{2}\left|\mathbf{E}_{2}(\theta, \phi)\right|^{2}+\left|\mathbf{E}_{1}(\theta, \phi)\right|\left|\mathbf{E}_{2}(\theta, \phi)\right| \sin \left(\arg \mathbf{E}_{1}(\theta, \phi)-\arg \mathbf{E}_{2}(\theta, \phi)\right), \\
R_{21}(\theta, \phi) & =\frac{1}{2}\left|\mathbf{E}_{1}(\theta, \phi)\right|^{2}+\frac{1}{2}\left|\mathbf{E}_{2}(\theta, \phi)\right|^{2}-\left|\mathbf{E}_{1}(\theta, \phi)\right|\left|\mathbf{E}_{2}(\theta, \phi)\right| \sin \left(\arg \mathbf{E}_{1}(\theta, \phi)-\arg \mathbf{E}_{2}(\theta, \phi)\right) .
\end{aligned}
$$




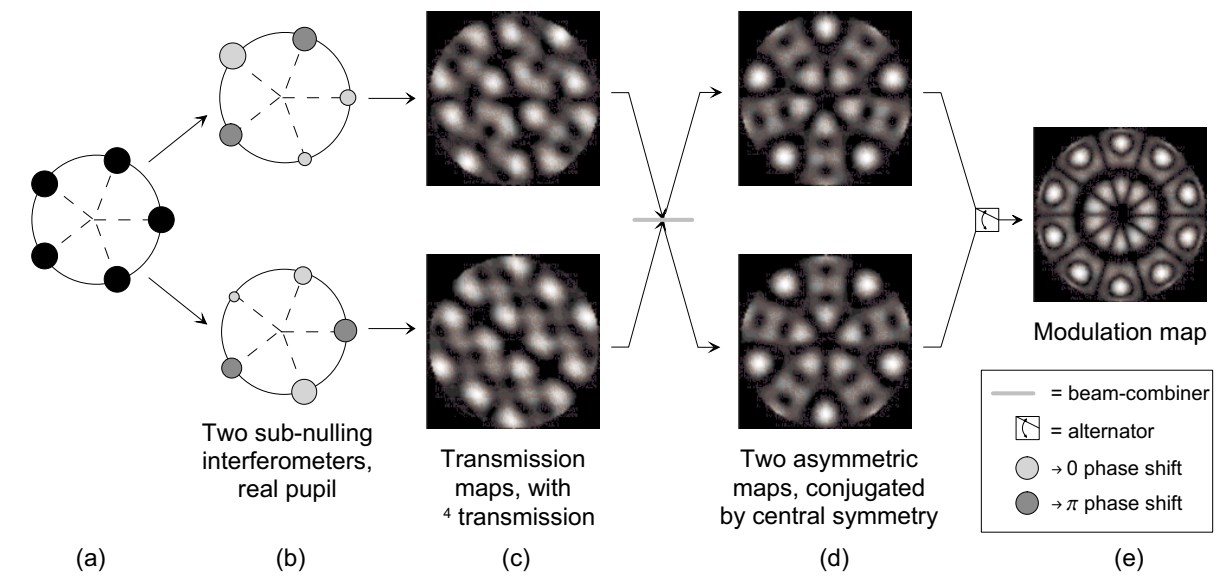

Figure 2. Principle of internal modulation. The outputs of two sub-interferometers are recombined on a lossless beamcombiner to produce the two output signals.

Now, if the entrance pupil of the two sub-interferometers are real, Eq. 1 gives $\mathbf{E}_{i}(\theta, \phi+\pi)=\overline{\mathbf{E}}_{i}(\theta, \phi)$ for $i=1,2$, where the bar denotes complex conjugation, so that $\arg \mathbf{E}_{i}(\theta, \phi+\pi)=-\arg \mathbf{E}_{i}(\theta, \phi)$ and thus $R_{12}(\theta, \phi+\pi)=$ $R_{21}(\theta, \phi)$. Therefore, an extended source with central symmetry such as the exozodiacal cloud has the same contribution in both outputs. The mean leakage of stellar light through the two transmission maps is also the same. On the other hand, the planet has two different contributions because when the planet lies on a bright zone in the first transmission map, it is located on a dark zone in the second map. By alternately detecting the two outputs on a common detector, we get a final signal where only the planetary part is modulated. The modulation map (Fig. 2e) shows which places on the sky are strongly modulated (bright zones), and where there is no modulation at all (dark zones).

A key advantage of internal modulation is that the switching between the two outputs can be done at a high frequency, so that there is now a clear distinction between the rapid modulation of the planetary signal and the slow fluctuations of the background. The two outputs will be continuously monitored by two single-pixel detectors to produce a maximum efficiency, and the maximum modulation frequency will be set by the detector read-out frequency. Another advantage of internal modulation is that in principle, the array need not be rotated any more. In practice, a slow rotation of the array will still be used in order to get a better spatial coverage.

In order to assess the efficiency of nulling configurations with internal modulation, the modulation efficiency is defined as the part of the incoming signal which is actually modulated and thus can be retrieved by synchronous demodulation. The expression of modulation efficiency reads:

$$
M E(\theta, \phi)=\frac{\left|R_{12}(\theta, \phi)-R_{21}(\theta, \phi)\right|}{\sum_{k=1}^{n} D_{k}^{2}}=\frac{2\left|\mathbf{E}_{1}(\theta, \phi)\right|\left|\mathbf{E}_{2}(\theta, \phi)\right|}{\sum_{k=1}^{n} D_{k}^{2}} \sin \left(\left|\arg \mathbf{E}_{1}(\theta, \phi)-\arg \mathbf{E}_{2}(\theta, \phi)\right|\right),
$$

where $D_{k}$ is the normalized diameter of telescope $k$ (normalization to 1 for the larger telescope). This definition of modulation efficiency allows an easy comparison between different configurations because it is independent of the number and size of telescopes, since the amplitude responses $\mathbf{E}_{i}(\theta, \phi)$ are normalized to the size of the larger telescope of the array.

Some configurations with internal modulation have already been proposed by Mennesson and Léger ${ }^{1}$ and by Karlsson and Mennesson. ${ }^{8}$ These include the so-called Robin Laurance configurations, which satisfy the following conditions:

- All telescopes are at equal distance from the beam combiner spacecraft to equalize the optical paths,

- All telescopes are in one plane (perpendicular to the line-of-sight) to reduce thermal coupling,

- The central transmission is proportional to $\theta^{4}$ (or $\theta^{6}$ ) to cancel the star light efficiently, 
- The only applied phase shift to each sub-interferometer is $\pi$ (real entrance pupils),

- All telescopes have the same diameter to reduce manufacturing costs.

Robin Laurance's configurations are referred to as $\operatorname{RL} x\left(m_{1}, m_{2}, \ldots, m_{n}\right)$, where $x$ is the number of sub-interferometers, $n$ the number of telescopes and $m_{i}$ the relative telescope size, starting with the largest one. The current baseline for the Darwin mission is the RL3(3,2,0,1,0,2) configuration with six telescopes arranged in a regular hexagonal configuration, as proposed by Karlsson and Mennesson. ${ }^{8}$ It achieves internal modulation between three Generalized Angel Crosses (GAC). It has a maximum modulation efficiency of 0.29, which means that in the best case, where the planet is located on a modulation maximum, only $29 \%$ of the planetary signal is actually modulated and can be retrieved by signal processing. The mean modulation efficiency on a field-of-view of $300 \times 300$ mas is of $12 \%$ for an array 25 meters in radius and a working wavelength of $10 \mu \mathrm{m}$. This is the relevant figure during the detection phase where the planet position is not known and the whole field-of-view has to be investigated. Note that a minimum separation of $20 \mathrm{~m}$ is required between the free-flying spacecrafts to reduce thermal coupling and to avoid collisions. Larger separations are preferred.

The main problem associated to this configuration, in addition to its rather low modulation efficiency, is the complexity of the optical recombination scheme to be implemented: it needs not less than nine beam-splitters with ratios of 5:4, 4:1 and 1:1, as well as nine beam-combiners and associated achromatic phase shifters to produce the three GAC outputs (see Karlsson and Mennesson ${ }^{8}$ ). Three more beam-combiners are needed to produce the six final outputs. Moreover, six detectors are needed for an optimum efficiency. Therefore, new configurations have to be found, yielding a higher modulation efficiency with a simpler recombination scheme. This would improve the reliability and decrease the cost of the mission.

\section{NEW CONFIGURATIONS WITH INTERNAL MODULATION}

Two main types of configurations have been considered so far: the circular ones by Mennesson and collaborators, and the linear ones mainly by Angel and Woolf. ${ }^{7}$ Both types of configurations can benefit from internal modulation. In order to achieve both a $\theta^{4}$ central transmission and internal modulation, linear (resp. circular) configurations need at least four (resp. five) telescopes, as proven by Absil. ${ }^{6}$

Circular configurations are very convenient for space interferometry: if the beam-combiner spacecraft is located at the center of the circle and the plane of the array perpendicular to the line-of-sight, the optical paths traveled by each light beam are equal, so that long delay lines are not required. A difficulty associated with linear configurations is that the light beams have to be reflected on neighboring telescopes in order to equalize the external optical delays (see Fig. 3). This could exaggeratedly constrain the control laws on their positions and attitudes.

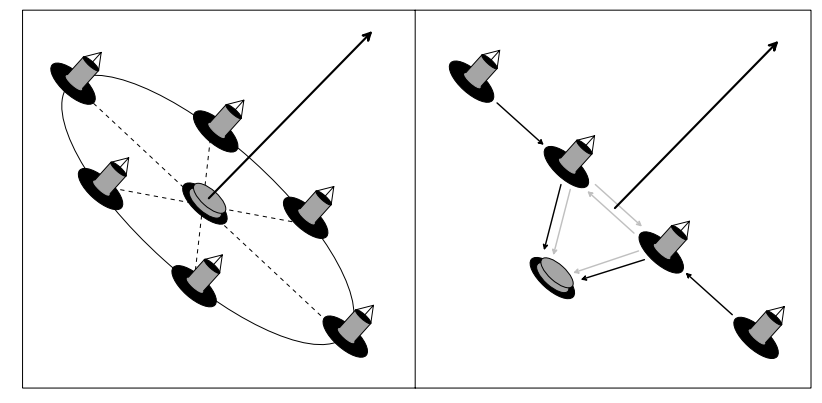

Figure 3. Optical path equalization in the cases of circular and linear configurations.

In the following paragraphs, we present a new circular configuration combining a high modulation efficiency and a rather simple recombination scheme. It is then compared with a very interesting linear configuration. 


\subsection{Circular configurations}

Circular configurations with five and six telescopes have been comprehensively studied by Absil. ${ }^{6}$ The best of them seems to be the so-called "bow-tie", also referred to as the "Liégeoise" configuration, with six telescopes in an irregular hexagonal shape (Fig. 4). This Robin Laurance configuration-RL2(1,1,0.707,0,0,0.707)—performs internal modulation between two identical sub-interferometers (GACs). Its maximum modulation efficiency reaches $65 \%$, that is, more than twice that of the original RL3 $(3,2,0,1,0,2)$ hexagon. Its mean modulation efficiency is still rather low $(13 \%)$ on a $300 \times 300$ mas field-of-view, but we can manage to get a high efficiency across the whole habitable zone of the target star by tuning the interferometer baseline (Fig. 5). The recombination scheme of the bow-tie (Fig. 4) is much easier to implement than the RL3 $(3,2,0,1,0,2)$ configuration, in that it involves only two 1:1 beam-splitters, six beam-combiners with associated achromatic phase shifters, and an additional beam-combiner to form the two final outputs. Two detectors are needed to ensure a maximum efficiency.
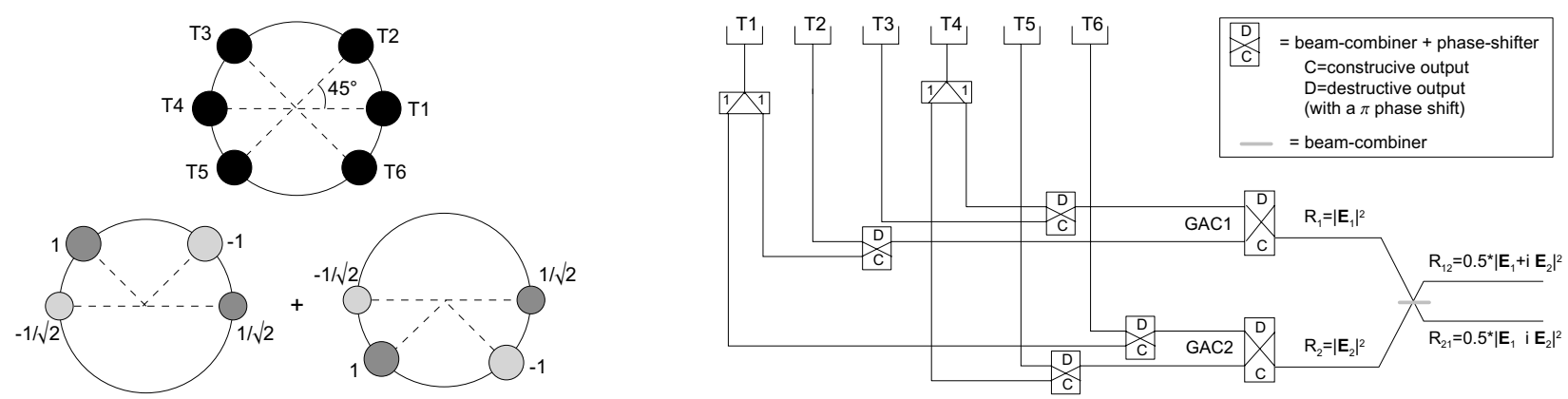

Figure 4. Left: The bow-tie configuration with its two sub-interferometers (Generalized Angel Crosses) and the relative signed amplitudes for each beam. Right: Recombination scheme for the bow-tie interferometer. The final outputs are characterized by their transmission maps $R_{12}$ and $R_{21}$. Note that one half of the intensity is lost at each beam-combiner because only one output is used.

The modulation map of this new configuration is presented in Fig. 5. It shows eight bright modulation maxima, each with a peak modulation efficiency of $65 \%$. With an array radius of $25 \mathrm{~m}$ and an observation wavelength of $10 \mu \mathrm{m}$, these maxima are located at 50 mas and 80 mas of the optical axis. This is also the typical angular separation between an Earth-like planet and its host star located at 10 pc. The right-hand side of Fig. 5 shows the result of a continuous rotation of the interferometer on the modulation map: we get two modulation crowns, with a peak efficiency of $65 \%$ for each. The coverage of the habitable zone is quite good, provided that the array radius is tuned to the desired target. A slow continuous rotation of the telescope array is not expected to increase the complexity of the mission, nor to require air important quantity of fuel.

With the bow-tie configuration, the time required to detect and characterize exoplanets by spectroscopy is reduced by a factor of four as compared to the RL3(3,2,1,0,1,0) configuration. This should allow to observe up to 500 main sequence stars during the mission lifetime instead of the 150 stars initially planned. This advantage adds to the relative simplicity of the recombination scheme, making the bow-tie a very good candidate for the final Darwin architecture.

\subsection{Linear configurations}

Linear configurations achieving internal modulation have been reviewed by Lawson et $\mathrm{al}^{9}{ }^{9}$ and have been further investigated by Absil. ${ }^{6}$ The most promising of them is presented in Fig. 6. This configuration is dubbed "LinEqual" because of its four equal-size telescopes. It achieves internal modulation between two irregular Degenerate Angel Crosses (DAC), which shapes have been chosen so that all telescopes have the same diameter. The recombination scheme of LinEqual is also presented in Fig. 6. It uses only four beam-combiners and associated achromatic phase shifters instead of six for the bow-tie. Note that the beam coming from the centre telescope of each DAC undergoes only one beam-combination, so that it is less attenuated than others. 

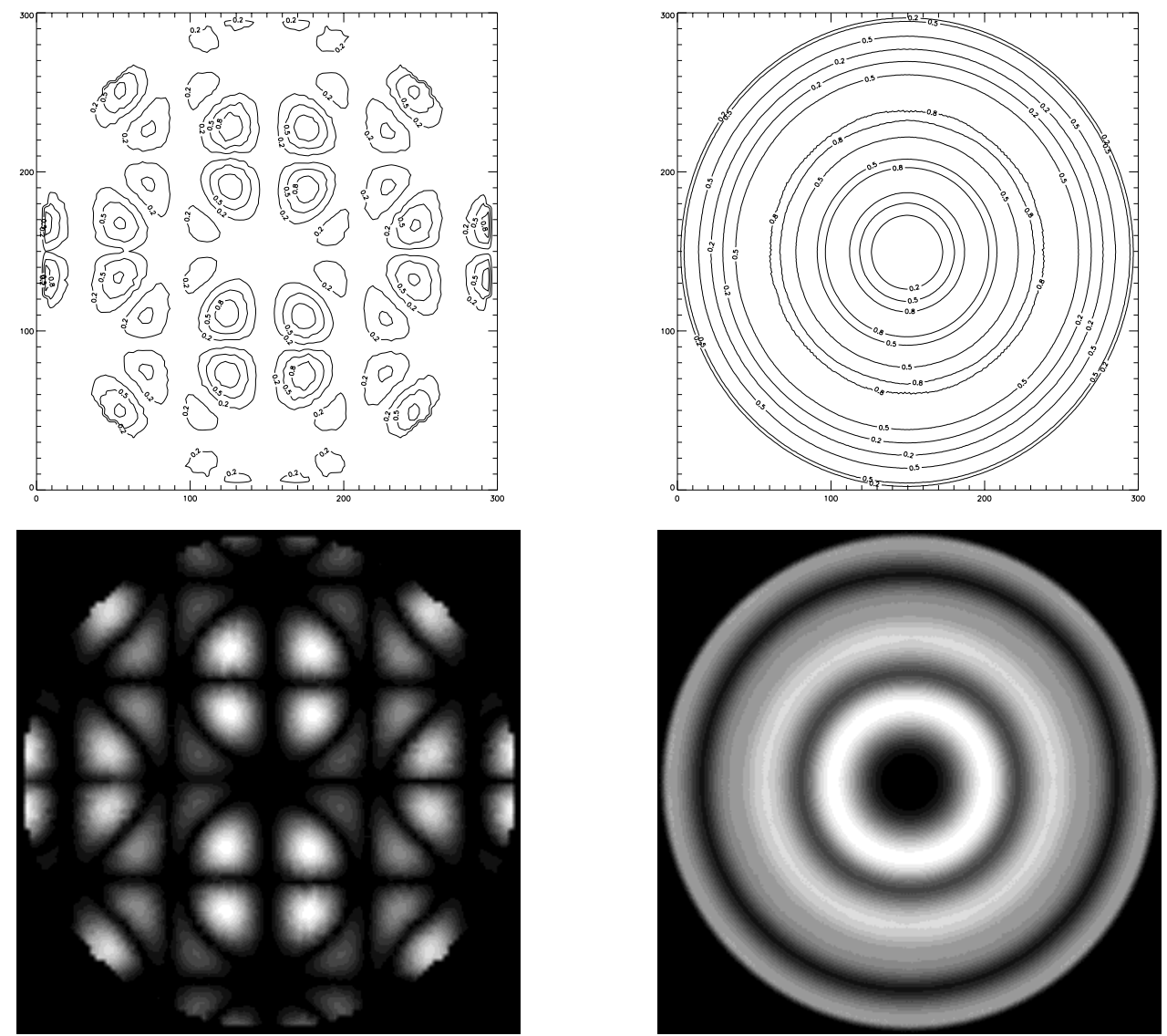

Figure 5. Left: Modulation map of the bow-tie interferometer over a $300 \times 300$ mas field-of-view for an array radius of $25 \mathrm{~m}$ and an observation wavelength of $10 \mu \mathrm{m}$ (isocontours at 20,50 and $80 \%$ of maximum). Right: Modulation map obtained by continuous rotation of the interferometer around its line-of-sight.
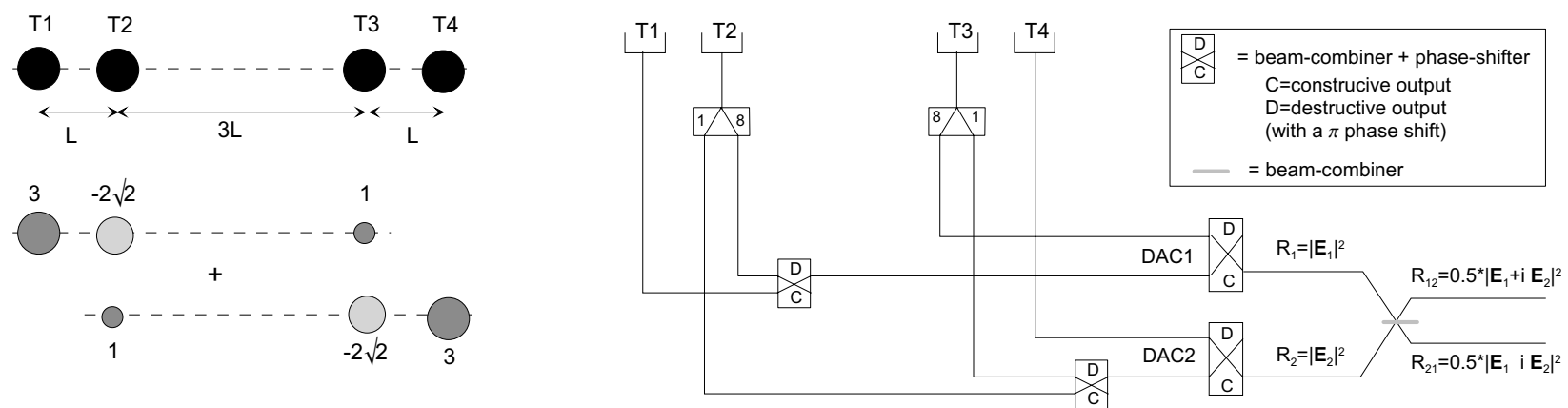

Figure 6. Left: The LinEqual configuration with its two sub-interferometers (Degenerated Angel Crosses) and the relative signed amplitudes for each beam. Right: Recombination scheme for the LinEqual interferometer. The final outputs are characterized by their transmission maps $R_{12}$ and $R_{21}$.

The modulation map of LinEqual is displayed in Fig. 7. The line-shaped map is characteristic of linear configurations. The maximum modulation efficiency of LinEqual is of $67 \%$, almost the same as for the bow-tie. Its mean modulation efficiency across the $300 \times 300$ mas field-of-view reaches $19 \%$, making LinEqual somewhat more efficient than the bow-tie during the detection phase, where the planet position is unknown and the whole 
field-of-view has to be investigated. A continuous rotation of the interferometer around its line of-sight is used to get a uniform sky coverage, as shown on the right-hand side of Fig. 7. In order to keep the separation between free-flying spacecrafts larger than 20 meters, an array length of 100 meters is needed. Assuming a working wavelength of $10 \mu \mathrm{m}$ as usual, the maximum of modulation efficiency is reached at about 60 mas from the optical axis, which makes this configuration appropriate for habitable planet detection around Sun-like stars at 10 pc.
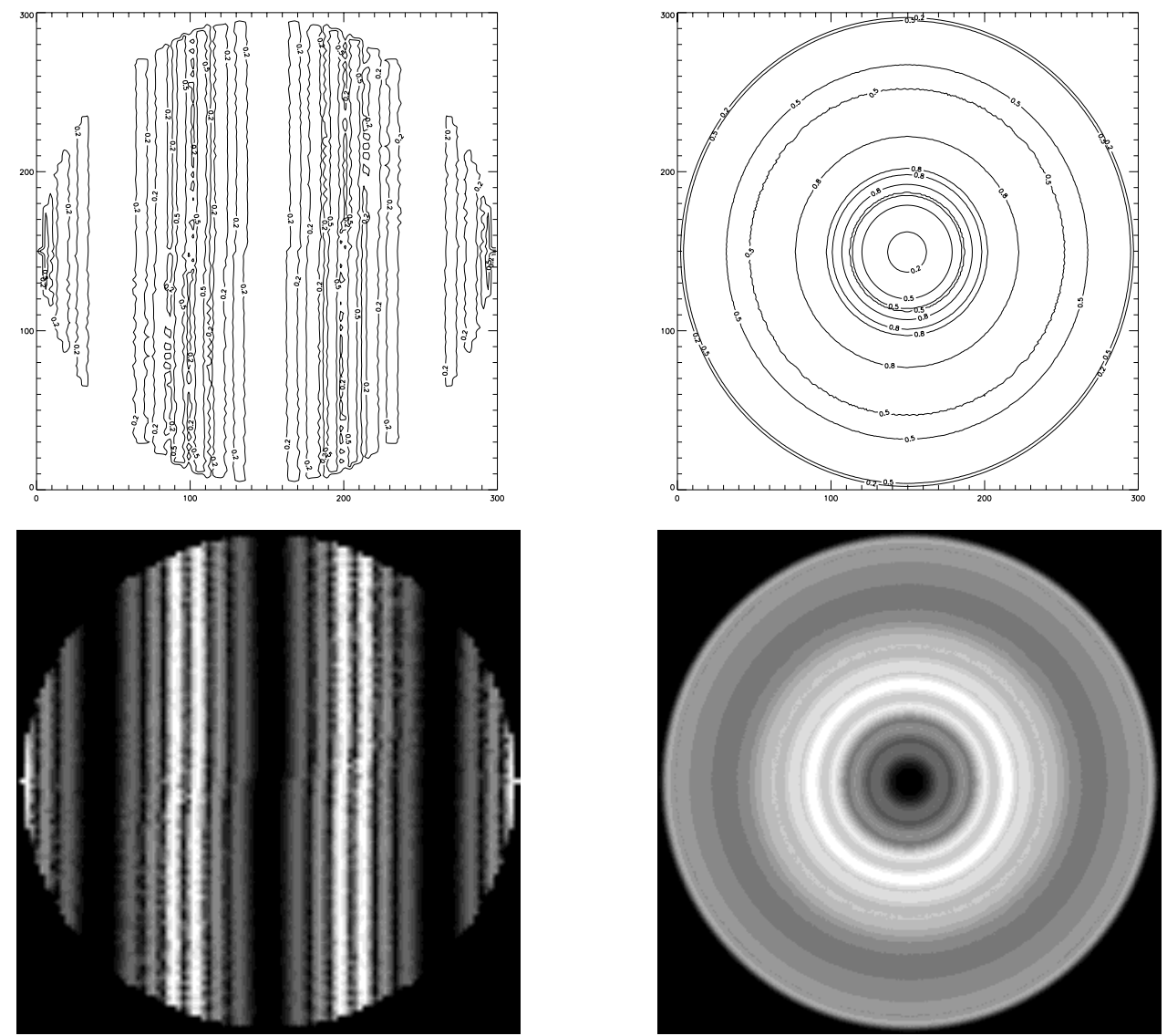

Figure 7. Left: Modulation map of the LinEqual interferometer over a $300 \times 300$ mas field-of-view for an array length of $100 \mathrm{~m}$ and an observation wavelength of $10 \mu \mathrm{m}$ (isocontours at 20,50 and 80\% of maximum). Right: Modulation map obtained by continuous rotation of the interferometer around its line-of-sight.

\section{INHERENT MODULATION}

We have seen how internal modulation uses two asymmetric transmission maps conjugated to one another to achieve a modulation of the planetary signal without modulating other signals. Keeping this basic principle, a new way to produce the conjugated maps has been found. The principle of inherent modulation is to combine a number of phase shifted beams, using two opposite sets of phase shifts to produce the two output signals (Fig. 8). The beam-combiner which was used to produce the two conjugated maps is not required any more. With inherent modulation, the two outputs are not formed simultaneously, but one after another: all the light is alternately sent to a set of phase shifting devices or to the other (Fig. 9). Therefore only one detector is needed, which reduces the detector noise by a factor of $\sqrt{2}$.

It is noteworthy that some of the achromatic phase shifts are different from 0 or $\pi$, and that they are generally not fractions of $\pi$. The entrance pupil of the interferometer is thus complex, which means that the amplitudes of the telescopes are multiplied by a set of complex coefficients $e^{i \phi_{k}}$ before recombination. A complex entrance 


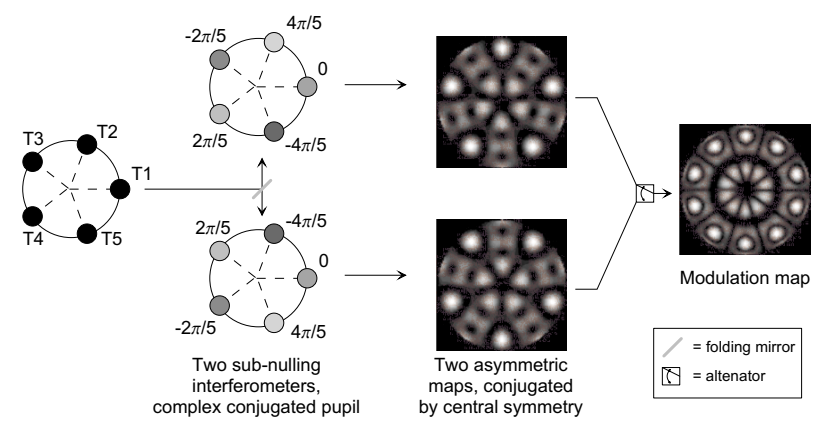

Figure 8. Principle of inherent modulation. Only one interferometer is used at a time.

pupil is a necessary — and almost sufficient - condition to get an asymmetric transmission map (Absil $\left.{ }^{6}\right)$. As in the case of internal modulation the two transmission maps are symmetric to each other with respect to their center, as proven by means of Eq. 1:

$$
\begin{aligned}
R_{1}(\theta, \phi+\pi) & =\left|\sum_{k=1}^{n} A_{k} e^{-i 2 \pi\left(L_{k} \theta / \lambda\right) \cos \left(\delta_{k}-\phi\right)} e^{i \phi_{k}}\right|^{2} \\
& =\left|\sum_{k=1}^{n} A_{k} e^{i 2 \pi\left(L_{k} \theta / \lambda\right) \cos \left(\delta_{k}-\phi\right)} e^{-i \phi_{k}}\right|^{2} \\
& =\left|\overline{\mathbf{E}}_{2}(\theta, \phi)\right|^{2}=R_{2}(\theta, \phi)
\end{aligned}
$$

A major advantage of inherent modulation is the relative simplicity of its recombination scheme. This is illustrated in Fig. 9, where the recombination schemes for a four-telescope linear array using internal and inherent modulations are compared. With inherent modulation, the beam-splitters are replaced by folding mirrors. Eight phase-shifting devices and three beam-combiners are used instead of six beam-combiners and associated phase shifters followed by another beam-combiner. The number of phase-shifting devices can sometimes be reduced if some of the $\phi_{i}$ are equal to some of the $-\phi_{j}$, which is the case in the example of Fig. 8 . Another example is the RL3 $3,2,1,0,1,0)$ configuration, which can be implemented on five beam-combiners instead of twelve.

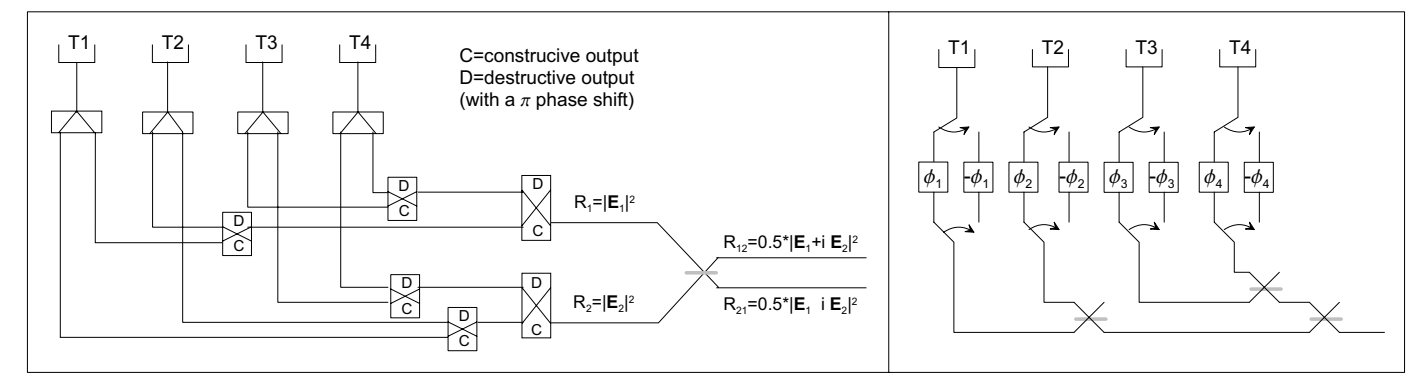

Figure 9. Recombination schemes for internal (left) and inherent (right) modulations in the case of a tour-telescope linen array. The number of beam-combinations is reduced from seven to there by using inherent modulation.

It can be demonstrated that all transmission maps obtained by published nulling techniques can be obtained without actually using sub-interferometers and modulation between sub-interferometers. In order to derive the sets of phase shifts and amplitudes for inherent modulation, starting from a known configuration with internal modulation, one calculates the contribution, in terms of amplitude and phase, from each input telescope to the 
output signal on the detector. The transposed transmission map is obtained by considering $-\pi / 2$ modulation rather than $+\pi / 2$ between the two sub-interferometers. All current configurations using inherent modulation are derived from known configurations with internal modulation. However, there is in principle no reason why a configuration using inherent modulation would necessarily have a counterpart based on modulation between sub-interferometers. Efforts are currently undertaken in order to identify such configurations.

\subsection{Circular configurations}

Circular arrays with a complex entrance pupil can achieve the necessary $\theta^{4}$ central transmission only if five telescopes or more are used (see $\mathrm{Absil}^{6}$ ). Recombining five telescopes at the same time forces us to use a third order recombination scheme (Mennesson and Mariotti ${ }^{5}$ ). This type of beam-combining scheme has a low efficiency: the recombination efficiency is of $5 / 8$, while it is equal to 1 for a four-telescope array. The recombination efficiency is the maximum constructive interference one can expect from a given recombination scheme. Therefore, the modulation efficiency of circular arrays with inherent modulation is generally low.

The simpler circular array with inherent modulation is the "Nils interferometer" shown in Fig. 8. Its modulation map has a very interesting shape allowing a good sky coverage without any array rotation. However, the maximum modulation efficiency is only $30 \%$, taking into account the fact that both outputs are only measured half of the time. Circular configurations with inherent modulation are not expected be significantly more efficient than this one, but their rather simple recombination schemes still make them good candidates for Darwin.

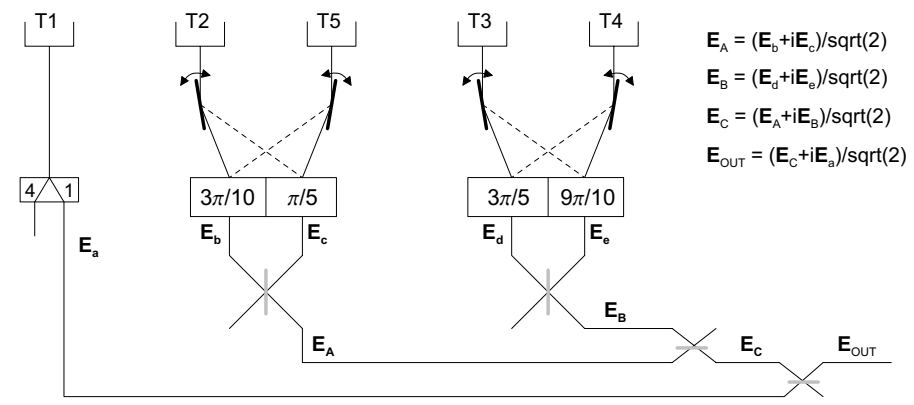

Figure 10. Third-order recombination scheme for the Nils interferometer. The flipping mirrors are used to send alternately the light to different phase-shifting devices. One half of the intensity is lost at each beam-combiner because only one output is used. The expressions of the electrical field after each beam-combiner are given in the top-right corner.

The recombination scheme of Nils is presented in Fig. 10. The achromatic phase-shifting devices are chosen so that the appropriate phase shifts are obtained after recombination, as proven by the expression of the output electrical field, including the $\pi / 2$ phase shift induced by the beam-combiners:

$$
\begin{aligned}
\mathbf{E}_{\mathrm{OUT}} & =\frac{1}{2 \sqrt{2}}\left(\mathbf{E}_{1}+e^{i \pi / 2}\left(e^{i 3 \pi / 10} \mathbf{E}_{2}+e^{i(\pi / 5+\pi / 2)} \mathbf{E}_{5}+e^{i \pi / 2}\left(e^{i 3 \pi / 5} \mathbf{E}_{3}+e^{i(9 \pi / 10+\pi / 2)} \mathbf{E}_{4}\right)\right)\right) \\
& =\frac{1}{2 \sqrt{2}}\left(\mathbf{E}_{1}+e^{i 4 \pi / 5} \mathbf{E}_{2}+e^{-i 4 \pi / 5} \mathbf{E}_{5}+e^{-i 2 \pi / 5} \mathbf{E}_{3}+e^{i 2 \pi / 5} \mathbf{E}_{4}\right)
\end{aligned}
$$

Another interesting circular configuration is the inherent bow-tie, with six equal-size telescopes positioned at $-\pi / 4,0, \pi / 4,3 \pi / 4, \pi$ and $5 \pi / 4$ with phase shifts of $3 \pi / 4,-2 \pi / 4, \pi / 4,-3 \pi / 4,2 \pi / 4$ and $-\pi / 4$. The transposed transmission map is obtained by applying the opposite phase shifts. This configuration has the same modulation map as the original bow-tie (Fig. 5), but the maximum efficiency is only of $33 \%$, i.e. half of the original one. The phase shifts could be implemented using for example a stack of phase-plates or by a combination of achromatic $\pi$-phase shifter and geometric delays. In the latter case, the positive phase shifts are implemented by a geometric delay of length corresponding to the required phase shift on a central wavelength (e.g. middle wavelength). The negative phase shifts are implemented in a similar way after having phase shifted the beams by $\pi$ using an 
achromatic phase shifter. The method builds on the principle that the chromatic error introduced by the delay lines belonging to the positive phase shifts are compensated by the errors of the delays lines of the negative phase shifts. This method does not achieve the required $10^{5}$ rejection rate across the whole 6-20 $\mu \mathrm{m}$ band, but could be implemented by dividing the domain into three smaller wavebands.

\subsection{Linear arrays}

In order to achieve a $\theta^{4}$ transmission with a complex-pupil linear array, four telescopes are needed at least $\left(\right.$ see $\left.\mathrm{Absil}^{6}\right)$. The second order recombination scheme used for four-telescopes arrays make them very attractive because the amount of light lost during the recombination process is much lower than for five-telescope arrays. Their efficiency is thus expected to be higher than for circular arrays. One of the best linear configurations is presented in table 1. It is composed of two big and two small telescopes (diameter ratio of 0.65), each one at equal distance of its neighbors. The maximum modulation efficiency of the LinOpt configuration is of $46 \%$

Table 1. Parameters for the LinOpt configuration.

\begin{tabular}{|l|c|c|c|c|}
\hline & T1 & T2 & T3 & T4 \\
\hline Relative diameter & $D_{1}=0.65$ & $D_{2}=1$ & $D_{3}=1$ & $D_{4}=1$ \\
Position & $L_{1}=0 \mathrm{~m}$ & $L_{2}=20 \mathrm{~m}$ & $L_{3}=40 \mathrm{~m}$ & $L_{4}=60 \mathrm{~m}$ \\
Phase shift & $\phi_{1}=-0.511$ & $\phi_{2}=3.142$ & $\phi_{3}=1.267$ & $\phi_{4}=4.920$ \\
\hline
\end{tabular}

and the mean efficiency of $17 \%$ on a $300 \times 300$ mas field-of-view. Its modulation map is presented in Fig. 11, showing two maxima of modulation located at 40 and 70 mas of the centre for an array length of $60 \mathrm{~m}$. The recombination scheme of LinOpt is presented on the right-hand side of Fig. 9. Eight phase-shifting devices and three beam-combiners are needed to produce the final output. A drawback of this configuration is the two different sizes for the telescopes, which could increase the manufacturing costs.

\section{CONCLUSION}

In this paper, new configurations with internal modulation have been investigated, leading to a simpler optical layout and increased performances: the maximum modulation efficiency reaches about $65 \%$, i.e. two times larger than for previous configurations. Inherent modulation, a new way to implement internal modulation, has been proposed, giving an even more simple layout provided that phase shifts different from $\pi$ can be achieved. This type of modulation is particularly adapted to linear configurations with four telescopes, yielding a maximum modulation efficiency of almost $50 \%$. These new configurations decrease the complexity of the mission by a factor of 2 , and increase the number of potential targets during the mission lifetime by a factor of 4 .

\section{ACKNOWLEDGMENTS}

This Research was supported through a European Community Marie Curie Fellowship. The author is solely responsible for the information communicated, published or disseminated. It does not represent the opinion of the Community. The Community is not responsible for any use that might be made of data appearing therein.

\section{REFERENCES}

1. B. Mennesson and A. Léger, "Direct detection and characterization of extrasolar planets: the Mariotti space interferometer." submitted to Icarus, 1999.

2. A. Léger, J.-M. Mariotti, B. Mennesson, M. Ollivier, J.-L. Puget, D. Rouan, and J. Schneider, "Could we search for primitive life on extrasolar planets in the near future? The DARWIN project," Icarus 123, p. 249, 1996.

3. R. Bracewell, "Detecting nonsolar planets by spinning an infrared interferometer," Nature 274, p. 780, 1978. 

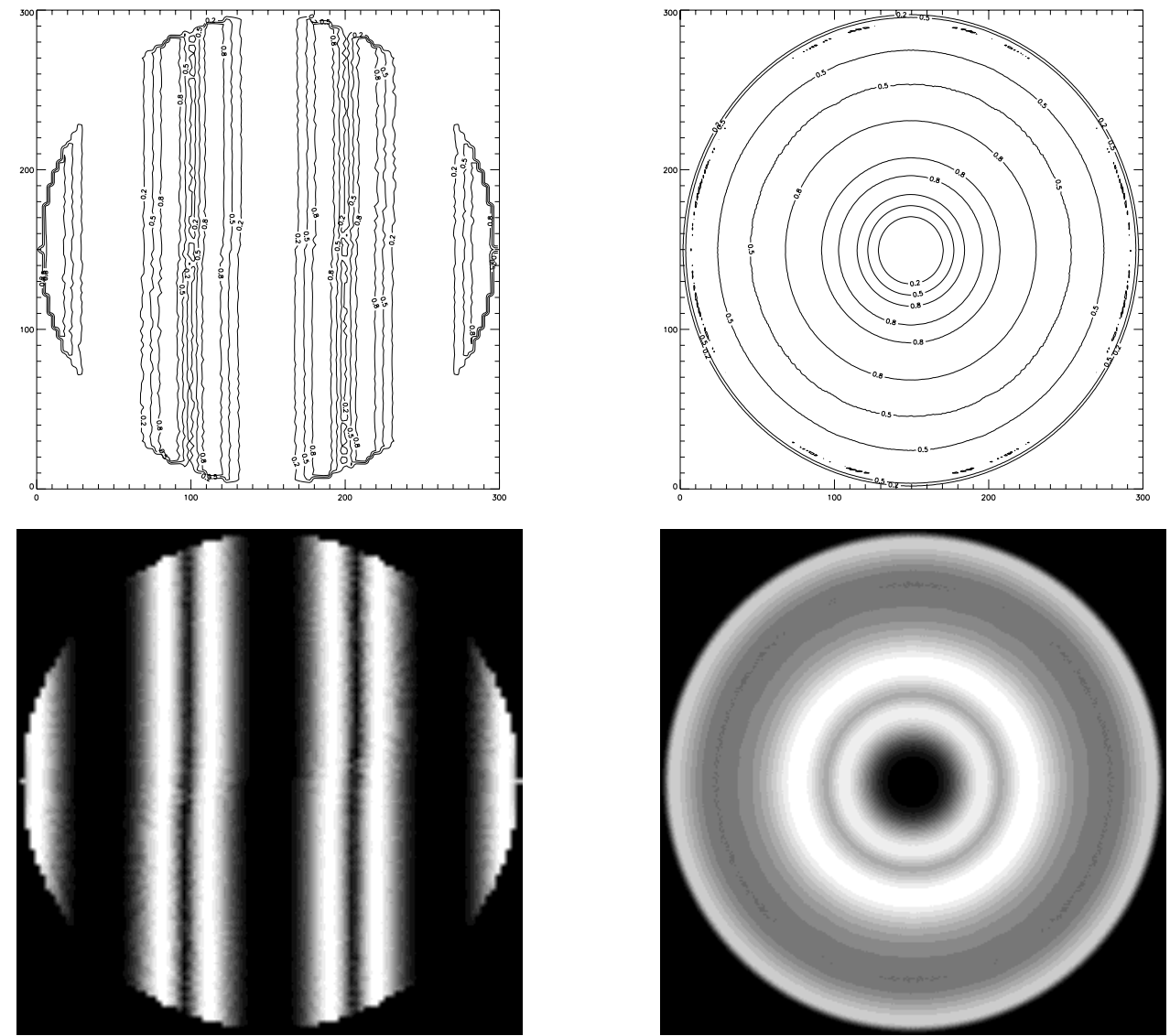

Figure 11. Left: Modulation map of the LinOpt interferometer over a $300 \times 300$ mas field-of-view for an array length of $60 \mathrm{~m}$ and an observation wavelength of $10 \mu \mathrm{m}$ (isocontours at 20,50 and 80\% of maximum). Right: Modulation map obtained by continuous rotation of the interferometer around its line-of-sight.

4. B. Mennesson, Interférométrie stellaire dans l'infrarouge thermique: observations d'environnements circumstellaires par optique guidée monomode et contributions à la mission spatiale DARWIN. PhD thesis, Université de Paris VII, December 1999.

5. B. Mennesson and J.-M. Mariotti, "Array configuration for a space infrared nulling interferometer dedicated to the search of earthlike extrasolar planets," Icarus 128, p. 202, 1997.

6. O. Absil, "Nulling interferometry with IRSI-Darwin: detection and characterization of Earth-like exoplanets," June 2001. End-of-studies dissertation for the degree of "Ingénieur physicien", Université de Liège, available at http://vela.astro.ulg.ac.be/themes/telins/harigs/research.html.

7. J. Angel and N. Woolf, "An imaging nulling interferometer to study extrasolar planets," ApJ 475, pp. 373-379, 1997.

8. A. Karlsson and B. Mennesson, "The Robin Laurance nulling interferometers," in Interferometry in Optical Astronomy, P. Lena and A. Quirrenbach, eds., Proc. SPIE 4006, pp. 871-880, 2000.

9. P. Lawson, P. Dumont, and M. Colavita, "Interferometer Designs for the Terrestrial Planet Finder," $A S P$ Conference Series 194, p. 423, 1999. 